\title{
MicroRNA-219-5p Promotes Tumor Growth and Metastasis of Hepatocellular Carcinoma by Regulating Cadherin 1
}

\author{
Jing Yang, ${ }^{1}$ Yuan-Yuan Sheng, ${ }^{2}$ Jin-Wang Wei, ${ }^{2}$ Xiao-Mei Gao, ${ }^{2}$ Ying Zhu, \\ Hu-Liang Jia $\mathbb{D}^{1},{ }^{1}$ Qiong-Zhu Dong $\mathbb{1}^{2},{ }^{2}$ and Lun-Xiu Qin $\mathbb{1}^{1}$ \\ ${ }^{1}$ Department of General Surgery, Huashan Hospital, Cancer Metastasis Institute, Fudan University, 12 Urumqi Road (M), \\ Shanghai 200040, China \\ ${ }^{2}$ Institutes of Biomedical Sciences, Fudan University, 131 Dong An Road, Shanghai 200032, China
}

Correspondence should be addressed to Hu-Liang Jia; jbl-1@163.com, Qiong-Zhu Dong; qzhdong@fudan.edu.cn, and Lun-Xiu Qin; qinlx@fudan.edu.cn

Received 16 January 2018; Accepted 19 March 2018; Published 15 May 2018

Academic Editor: Dimitrios P. Bogdanos

Copyright (C) 2018 Jing Yang et al. This is an open access article distributed under the Creative Commons Attribution License, which permits unrestricted use, distribution, and reproduction in any medium, provided the original work is properly cited.

MicroRNAs play significant roles in the development of cancer and may serve as promising therapeutic targets. In our previous work, miR-219-5p was identified as one of the important metastasis-related microRNAs in HCC. Here we demonstrated that miR219-5p expression was elevated in HCC tissues and was associated with vascular invasion and dismal prognosis. In multivariate analysis, miR-219-5p was identified as an independent prognostic indicator for HCC patients. Functional mechanism analyses showed that miR-219-5p promoted HCC cell proliferation and invasion in in vitro, as well as in vivo, tumor growth and metastasis in nude mice models bearing human HCC tumors. In addition, cadherin 1 (CDH1) was revealed to be a downstream target of miR-219-5p in HCC cells. In conclusion, miR-219-5p promotes tumor growth and metastasis of HCC by regulating CDH1 and can serve as a prognostic marker for HCC patients.

\section{Introduction}

Hepatocellular carcinoma (HCC) is one of the most common causes of cancer-related deaths worldwide, with high incidence of tumor recurrence and metastasis [1]. Identification of molecular markers plays a critical role in predicting the clinical outcome and promoting individual therapies for patients with HCC $[2,3]$.

MicroRNAs (miRNAs) have been implicated in regulation of pathogenesis of human tumors and could be potential biomarkers for diagnosis and prognosis $[4,5]$. Recent studies have demonstrated that miRNAs participate in diverse human cancers processes including cell differentiation, proliferation, and apoptosis, as well as invasion and metastasis. For instance, miR-125 is a tumor suppressor that can decrease cell proliferation and metastasis through suppressing LIN28B expression in HCC [6], while miR-122a exerts tumor promoting effects on HCC by p53-dependent way [7]. Thus, cancer-specific miRNAs might be promising targets for cancer therapy [8].
Recently, miRNAs are demonstrated to function as critical regulators of cancer invasion and metastasis [9]. In our previous work, miR-219-5p is identified as one of the significant metastasis-related miRNAs in HCC [10]. However, little is known of the possible mechanism of miR-219-5p involved in HCC metastasis. In the present study, we found that miR-219-5p was upregulated in HCC tissues, was related to overall survival (OS) time of HCC patients, and promoted the proliferation and metastasis of HCC cells via downregulating $\mathrm{CDH} 1$. These results provide a clear understanding of the underlying mechanism by which miR-219-5p promotes HCC metastasis.

\section{Materials and Methods}

2.1. Clinical Tissue and Cell Culture. HCC tissues were obtained from patients who are treated with surgical resection in Huashan Hospital, Fudan University, and each patient had specific clinical-pathological information. 
Before surgical operations and collections of clinical tissues, all individuals wrote informed consent.

Human HCC cell lines Hep3B, Huh7, HepG2, MHCC97H, and HCCLM3 were cultured in DMEM (Gibco) with $10 \% \mathrm{FBS}$. And they were propagated at $37^{\circ} \mathrm{C}$ in $5 \% \mathrm{CO}_{2}$.

2.2. Cell Transfection. HepG2 and MHCC-97H cells were transfected with miR-219-5p mimic (50 nM) and miR-219$5 \mathrm{p}$ antagomir $(400 \mathrm{pmol} / \mathrm{ml})$ according to the manufacturer instructions. miR-219-5p mimic, antagomir, and their corresponding negative controls were purchased from Ribobio (Shanghai, China).

2.3. RNA Extraction and Quantitative Reverse TranscriptionPolymerase Chain Reaction ( $q R T-P C R)$. RNA of samples was obtained by TRIzol reagent (Invitrogen, USA). Then, we reversely transcripted RNA into cDNA according to the instruction of PrimeScript RT Master Mix and Mir-X miRNA First-Strand Synthesis Kit (TaKaRa, Shanghai, China). Next, cDNA was quantified by application of SYBR Premix Ex Taq II (TaKaRa) with gene-targeted or miR-specific primers. We applied the delta-delta $\mathrm{Ct}$ method to conduct quantification as well as calculation of the relative expression of each mRNA or miRNA. Primer sequences are listed in Table S1. Each sample was carried out three times.

2.4. Cell Proliferation Assay. The cell proliferation assay was conducted with Counting Kit-8 (CCK-8) (Tongren, Shanghai, China). 5000 cells (transfected with miR-219-5p mimic, miR-ctrl, miR-219-5p antagomir, antagomir NC) were planted in 96 -well plates. Then $10 \%$ CCK-8 solution was added. The absorbance of each sample was assessed by a microplate reader set at $450 \mathrm{nM}$. Each sample was performed three times.

2.5. Cell Cycle Analysis and Apoptosis Assay. Cell cycle analysis was conducted with each sample fixed into $70 \%$ ethanol at $4^{\circ} \mathrm{C}$. We added Propidium iodide (PI) and RNase to samples according to manufactures' instructions (Beyotime, Shanghai, China). After staining, cells were measured by flow cytometry (BD Bioscience, MA, USA). We analyzed results by Cell Quest software (BD Biosciences). Apoptosis assay was conducted by samples bound with Annexin V-FITC and 7-AAD according to the manufacturers' instructions (BD Bioscience). Then we analyzed samples by means of flow cytometry as described above. Each sample was replicated in triplicate.

2.6. Dual-Luciferase Reporter Assay. The wild-type sequence containing the predicted target sites of miR-219-5p in the $3^{\prime}$ UTR of CDH1 mRNA was synthesized by JIELI corporation (Shanghai, China). We mutated the target sites from CUCCAC to GACCGA. After plasmid transfection, luciferase activities were assessed according to the manufacturer's instruction (Promega, USA). All samples were independently repeated three times.
2.7. Western Blotting. Samples were obtained with RIPA lysis buffer added with protease inhibitors. After quantification with bicinchoninic acid (BCA) assay (Weiao, Shanghai, China), we separated each protein through 10\% SDS-PAGE and then moved them onto PVDF membranes (Millipore, USA). Then, samples were blocked with 5\% nonfat milk. After incubation with primary antibodies against GAPDH and CDH1 (Cell Signaling Technology, Danvers, MA, USA) and secondary antibodies, protein levels were detected with ImageQuant ${ }^{\mathrm{TM}}$ LAS 4000 (GE Healthcare Life Sciences). Each sample was analyzed three times.

2.8. Cell Migration and Invasion Assay. The methods of cell migration and invasion assays were constructed as previously described [11].

2.9. Animal Model. Subcutaneous HCC model was established by injecting $5 \times 10^{6}$ MHCC-97H cells (transfected with antagomir NC or antagomir miR-219-5p) into BALB/c nude mice (Shanghai SLAC Laboratory Animal Co.). And after 6 weeks, the tumor of each group was isolated. To establish in vivo tumor metastasis model, we transplanted tumors tissues $\left(1 \sim 2 \mathrm{~mm}^{3}\right)$ from the above subcutaneous HCC model to the livers of $\mathrm{BALB} / \mathrm{c}$ mice. After 6 weeks, the tumors and lung tissues were obtained.

Tumor size was measured twice weekly with a calliper and the volume was calculated in $\mathrm{mm}^{3}$.

2.10. Statistical Analysis. Data was reported as mean $\pm \mathrm{SD}$. Data analysis was conducted by IBM SSPS Statistics Version 22. $X^{2}$ and $t$-test were applied to measure differences between groups. Results were determined to be statistically significant when $P<0.05$.

\section{Results}

3.1. miR-219-5p Upregulation Is Associated with Metastasis and Dismal Prognosis of HCC. We analyzed the expression levels of miR-219-5p in 191 paired HCC tissues and corresponding noncancerous liver tissues by using qRT-PCR and found that miR-219-5p was significantly increased in HCC tissues compared with the nontumor liver tissues $(P<$ 0.001) (Figure 1(a)). The expression levels of miR-219-5p were remarkably higher in HCC patients with metastasis in comparison to those without metastasis $(P<0.001)$ (Figure 1(b)). To further validate the role of miR-219-5p in HCC metastasis, we analyzed miR-219-5p in various HCC cell lines with different metastatic potentials and found that miR-219-5p levels in the HCC cells with high metastatic potentials were higher than those nonmetastatic cell lines (Supplementary Figure 1(A)). These results indicated that miR-219-5p upregulation is correlated with HCC metastasis.

Moreover, elevated miR-219-5p expression was found to be correlated with vascular invasion $(P=0.003)$ and worse differentiation $(P=0.011)$ of liver tumor, as well as severe liver cirrhosis $(P<0.001)$ (Table 1). Kaplan-Meier analysis showed that miR-219-5p overexpression was associated with poorer overall survival and higher recurrence 


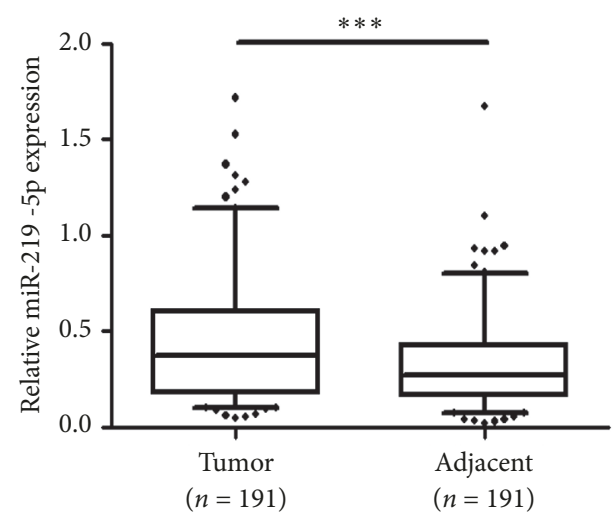

(a)

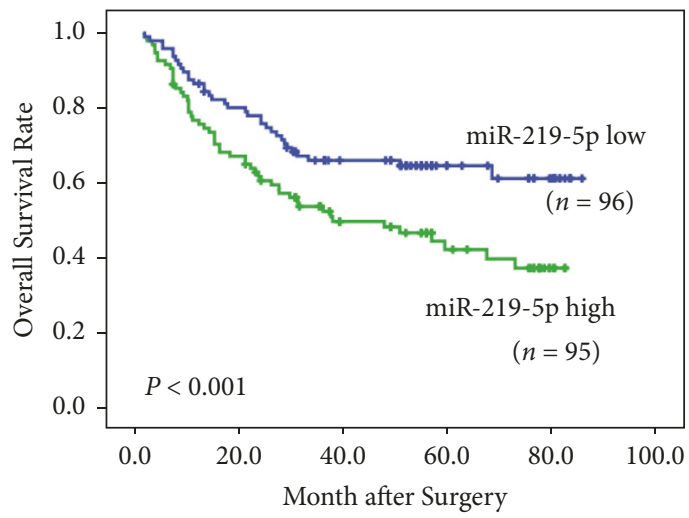

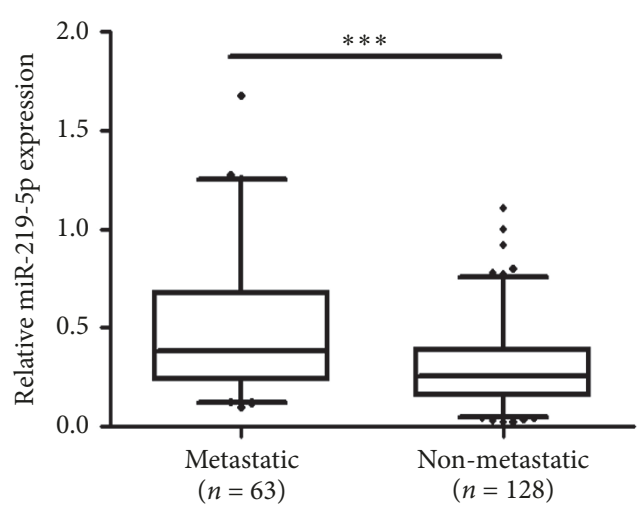

(b)

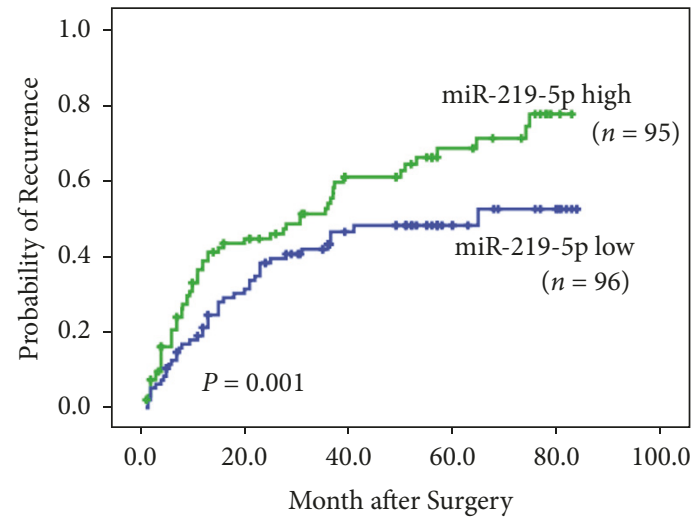

(c)

FIGURE 1: The association of miR-219-5p upregulation with metastasis and prognosis of HCC. (a) Relative expressions of miR-219-5p in 191 paired liver cancer tissues and paracancerous tissue samples. (b) The comparison of miR-219-5p levels between metastatic and nonmetastatic HCC tissues. Patients with high miR-219-5p level had a trend of worse overall survival (c) and significantly high recurrence rates compared with those with low miR-219-5p (d). Data are shown as mean \pm SD. ${ }^{* * *} P<0.001$ versus the control.

rates of patients after curative HCC resection (Figure 1(c)). Univariate analysis showed that miR-219-5p, tumor size, tumor encapsulation, and vascular invasion were related to overall survival (OS) (Table 2); miR-219-5p, HBsAg, tumor size, vascular invasion, and tumor number were associated with HCC recurrence (Table 3). Multivariate analysis showed that miR-219-5p, vascular invasion, and tumor size were independent prognostic indicators for overall survival and tumor recurrence. Therefore, these results suggested that miR-219-5p upregulation can be a predictor of metastasis and dismal prognosis of HCC patients.

\subsection{The Effects of miR-219-5p on In Vitro Proliferation and} Invasion of HCC Cells. To investigate the biological significance of miR-219-5p, we treated human HCC cell lines with miR-219-5p mimic or antagomir that would lead to different expression levels of miR-219-5p. Upregulation of miR-219$5 \mathrm{p}$ in HepG2, which had a low endogenous expression level, by miR-219-5p mimic induced significant increases in the abilities of proliferation (Figure 2(a); Supplementary Figure 2(A)). On the other hand, knockdown of miR-219$5 p$ in MHCC-97H (with a high endogenous miR-219-5p level) by miR-219-5p antagomir (Supplementary Figure 2(A)) significantly inhibited the proliferation of cells (Figure 2(a)). What is more, the cell cycle distribution analysis showed that the cell number in G1 phase of HepG2 cells treated with miR-219-5p mimic was obviously decreased, and the cell number in $S$ phase was increased compared with the ctrl. Cell cycle arrest at the G1 to S transition was found in MHCC-97H cells after treated with miR-219-5p antagomir (Figure 2(b); Supplementary Figure 2(B)). Furthermore, miR219-5p mimic transfection significantly suppressed the apoptosis of HepG2 cells compared with ctrl, while miR-219-5p downregulation induced by miR-219-5p antagomir markedly promoted the apoptosis of MHCC-97H cells (Figure 2(c)). Next, we performed transwell assays to evaluate the invasion and migration abilities of HepG2 and MHCC-97H cells. Results showed that miR-219-5p upregulation significantly enhanced the migration and invasion abilities of HepG2, and miR-219-5p knockdown induced by miR-219-5p antagomir led to reduced number of migrated and invaded cells (Figure 2(d); Supplementary Figure 2(C)). Taken together, these data suggested that miR-219-5p can promote the proliferation, cell cycle transition of G1 into S phase, antiapoptotic potentials, and metastatic phenotype of HCC cells. 
TABLE 1: Relationship between miR-219-5p level and clinicopathologic features.

\begin{tabular}{|c|c|c|c|}
\hline \multirow[b]{2}{*}{ Variables } & \multicolumn{2}{|c|}{ miR-219-5p expression } & \multirow[b]{2}{*}{$P$ value } \\
\hline & $\begin{array}{c}\text { Low } \\
(n=96)\end{array}$ & $\begin{array}{l}\text { High } \\
(n=95)\end{array}$ & \\
\hline \multicolumn{4}{|l|}{ Sex } \\
\hline Female & 18 & 13 & \multirow{2}{*}{0.342} \\
\hline Male & 78 & 82 & \\
\hline \multicolumn{4}{|l|}{ Age (years) } \\
\hline$\geq 50$ & 32 & 30 & \multirow{2}{*}{0.796} \\
\hline$<50$ & 64 & 65 & \\
\hline \multicolumn{4}{|l|}{ HBV status } \\
\hline Yes & 94 & 43 & \multirow{2}{*}{0.599} \\
\hline No & 9 & 15 & \\
\hline \multicolumn{4}{|l|}{ Cirrhosis } \\
\hline Yes & 16 & 55 & \multirow{2}{*}{$<0.001$} \\
\hline No & 80 & 40 & \\
\hline \multicolumn{4}{|c|}{$\operatorname{AFP}(n g / m L)$} \\
\hline$>20$ & 64 & 63 & \multirow{2}{*}{0.959} \\
\hline$\leq 20$ & 32 & 32 & \\
\hline \multicolumn{4}{|c|}{ Tumor size $(\mathrm{cm})$} \\
\hline$>5$ & 40 & 43 & \multirow{2}{*}{0.616} \\
\hline$\leq 5$ & 56 & 52 & \\
\hline \multicolumn{4}{|c|}{ Tumor number } \\
\hline Multiple & 4 & 9 & \multirow{2}{*}{0.145} \\
\hline Single & 92 & 86 & \\
\hline \multicolumn{4}{|c|}{ Tumor encapsulation } \\
\hline Yes & 52 & 45 & \multirow{2}{*}{0.347} \\
\hline No & 44 & 50 & \\
\hline \multicolumn{4}{|c|}{ Vascular invasion } \\
\hline Yes & 31 & 51 & \multirow{2}{*}{0.003} \\
\hline No & 65 & 44 & \\
\hline \multicolumn{4}{|c|}{ Tumor differentiation } \\
\hline $\mathrm{I} \sim \mathrm{II}$ & 65 & 48 & \multirow{2}{*}{0.011} \\
\hline III IV & 31 & 47 & \\
\hline
\end{tabular}

3.3. Effects of miR-219-5p on In Vivo Tumor Growth and Lung Metastasis of HCC Xenografts. To further validate promoting roles of miR-219-5p in HCC progression, we established HCC xenografts models by subcutaneous implantation of MHCC-97H cells (transfected with miR-219-5p antagomir or antagomir NC). The average tumor volume of the miR219-5p antagomir-treated group was obviously smaller than that of antagomir NC group $(P<0.05)$ (Figures 3(a) and $3(\mathrm{~b})$ ). To further validate its effect on the lung metastasis of HCC, the tumor tissues $\left(1-2 \mathrm{~mm}^{3}\right)$ were obtained from subcutaneous xenografts to establish orthotopic implantation models of nude mice. The average volume of orthotopic tumors in the miR-219-5p antagomir group was significantly smaller than that in the antagomir NC group $(P<0.05)$ (Figure 3(c)). Moreover, the total number of lung metastases in the miR-219-5p antagomir group was decreased compared with the antagomir NC $(P<0.01)$ (Figure $3(\mathrm{~d}))$. These results suggested that miR-219-5p plays a crucial role in promoting in vivo tumor growth and lung metastasis of HCC.

3.4. CDH1 Is a Direct Target of microRNA-219-5p. Next, we searched for putative target genes of miR-219-5p in microRNA.org. We identified CDH1 as a direct target of miR219-5p and the potential binding sequence in CDH1 $3^{\prime}$ UTR (Figure 4(a)). We carried out a dual-luciferase reporter assay to prove that $\mathrm{CDH} 1$ is a direct target of miR-219$5 \mathrm{p}$. The reporter vector containing wild-type (CDH1-WT) or mutated-type binding sequence (CDH1-MT) was transfected into HEK293T cells along with miR-219-5p mimic or ctrl. Results showed that cotransfection of miR-219-5p with CDH1-WT, rather than with CDH1-MT, resulted in a significant decrease in luciferase activity compared with ctrl group $(P<0.05)$ (Figure 4(b)). To further validate the influence of miR-219-5p on CDH1, we overexpressed miR-219-5p in HepG2 cells and knocked down miR-219-5p in MHCC-97H cells, finding that miR-219-5p upregulation led to a significant decrease of $\mathrm{CDH} 1$ expression at both mRNA and protein levels $(P<0.01)$. And, miR-219-5p knockdown resulted in enhanced CDH1 expression $(P<$ 0.01 ) (Figures 4(c) and 4(d)). Moreover, the linear regression analysis showed a negative relevance between miR-219-5p and CDH1 in HCC tissues $\left(R^{2}=0.4225 ; P<0.001\right)$ (Figure 4(e)). These suggested that miR-219-5p is closely associated with negative regulation of $\mathrm{CDH1}$ and $\mathrm{CDH} 1$ is a direct target of miR-219-5p.

\section{Discussion}

Metastatic relapse remains one of the major reasons for the dismal prognosis of HCC, which is a complicated process including cell adhesion, migration, and getting to target organs. Many molecules have been determined to be related to HCC metastasis [12]. However, the mechanism of HCC metastasis is not fully understood yet. Thus, characterizing the metastasis-related molecules and signaling pathways may provide more clues to the understanding of HCC metastasis. The clinical relevance and biological functions of miRNAs expression have been confirmed in various human solid tumors [13]. Thus, miRNAs were identified as superior molecular markers. Recently, an increasing number of studies have reported the indispensable roles of miRNAs in HCC [14-16]. In our previous study, miR-219-5p was found to be a promoter for HCC metastasis [10]. However, some studies demonstrated inconsistent results in other kinds of cancers. For example, miR-219-5p was reported to function as a tumor suppressor in colorectal and gastric cancers $[17,18]$. The real reason is not clear. These results stimulate us to investigate the role of miR-219-5p in regulating aggressive phenotype of HCC cells.

In the present study, we found that miR-219-5p expression levels were remarkably upregulated in HCC tissues compared with the nontumor liver tissues, and high miR-219-5p levels were significantly associated with metastasis and dismal prognosis of HCC. Using gain- and loss-functional analyses, 
TABLE 2: Univariate and multivariate analyses of factors associated with overall survival (OS) inpatients with hepatocellular carcinoma (HCC).

\begin{tabular}{|c|c|c|c|c|}
\hline \multirow{3}{*}{ Features } & \multicolumn{4}{|c|}{ Overall survival } \\
\hline & \multirow{2}{*}{ Univariate $P$} & \multicolumn{3}{|c|}{ Multivariate } \\
\hline & & HR & $95 \%$ CI & $P$ \\
\hline \multicolumn{5}{|l|}{ 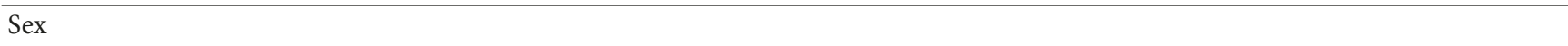 } \\
\hline Male versus female & 0.537 & & & NA \\
\hline \multicolumn{5}{|l|}{ Age } \\
\hline$>50$ versus $\leq 50$ & 0.404 & & & NA \\
\hline \multicolumn{5}{|l|}{ HBsAg } \\
\hline Positive versus negative & 0.112 & & & NA \\
\hline \multicolumn{5}{|l|}{ AFP } \\
\hline $20 \mathrm{ng} / \mathrm{ml}$ versus $\leq 20 \mathrm{ng} / \mathrm{ml}$ & 0.069 & & & NA \\
\hline \multicolumn{5}{|l|}{ Liver cirrhosis } \\
\hline Yes versus no & 0.307 & & & NA \\
\hline \multicolumn{5}{|l|}{ Tumor size } \\
\hline$>5 \mathrm{~cm}$ versus $\leq 5 \mathrm{~cm}$ & $<0.001$ & 2.620 & $1.675 \sim 4.099$ & $<0.001$ \\
\hline \multicolumn{5}{|l|}{ Tumor encapsulation } \\
\hline Yes versus no & 0.017 & 1.084 & $0.666 \sim 1.764$ & 0.746 \\
\hline \multicolumn{5}{|l|}{ Tumor number } \\
\hline Multiple versus single & 0.065 & & & \\
\hline \multicolumn{5}{|l|}{ Vascular invasion } \\
\hline Yes versus no & 0.001 & 1.833 & $1.179 \sim 2.848$ & 0.007 \\
\hline \multicolumn{5}{|l|}{ Tumor differentiation } \\
\hline I $\sim$ II versus III $\sim$ IV & 0.113 & & & \\
\hline \multicolumn{5}{|l|}{$\operatorname{miR}-219-5 p$} \\
\hline High versus low & 0.007 & 1.689 & $1.433 \sim 3.903$ & 0.036 \\
\hline
\end{tabular}

TABLE 3: Univariate and multivariate analyses of factors associated with recurrence in patients with hepatocellular carcinoma (HCC).

\begin{tabular}{|c|c|c|c|c|}
\hline \multirow{3}{*}{ Features } & \multicolumn{4}{|c|}{ Recurrence } \\
\hline & \multirow{2}{*}{ Univariate $P$} & \multicolumn{3}{|c|}{ Multivariate } \\
\hline & & HR & $95 \% \mathrm{CI}$ & $P$ \\
\hline Sex & & & & NA \\
\hline Male versus female & 0.331 & & & NA \\
\hline Age & & & & NA \\
\hline$>50$ versus $\leq 50$ & 0.832 & & & NA \\
\hline HBsAg & & & & NA \\
\hline Positive versus negative & 0.043 & 0.761 & $0.392 \sim 1.476$ & 0.419 \\
\hline AFP & & & & NA \\
\hline $20 \mathrm{ng} / \mathrm{ml}$ versus $\leq 20 \mathrm{ng} / \mathrm{ml}$ & 0.615 & & & NA \\
\hline Liver cirrhosis & & & & NA \\
\hline Yes versus no & 0.065 & & & NA \\
\hline Tumor size & & & & NA \\
\hline$>5 \mathrm{~cm}$ versus $\leq 5 \mathrm{~cm}$ & $<0.001$ & 1.744 & $1.274 \sim 2.388$ & $<0.001$ \\
\hline Tumor encapsulation & & & & NA \\
\hline Yes versus no & 0.671 & & & NA \\
\hline Tumor number & & & & NA \\
\hline Multiple versus single & 0.010 & 1.579 & $0.751 \sim 3.318$ & 0.228 \\
\hline Vascular invasion & & & & NA \\
\hline Yes versus no & 0.014 & 1.870 & $1.205 \sim 2.902$ & 0.005 \\
\hline Tumor differentiation & & & & NA \\
\hline I II versus III IV & 0.539 & & & NA \\
\hline miR-219-5p & & & & NA \\
\hline High versus low & 0.014 & 1.663 & $1.072 \sim 2.577$ & 0.023 \\
\hline
\end{tabular}

Abbreviations. HBsAg: hepatitis B surface antigen; HR: hazard ratio; CI: confidence interval. 


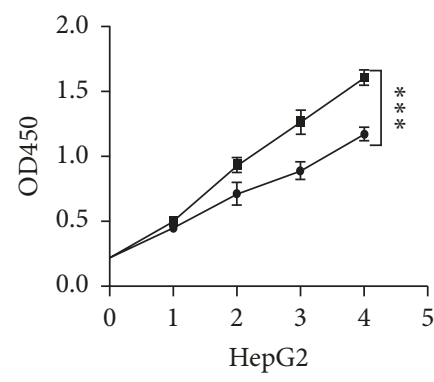

$\rightarrow$ miR-219-5p ctrl $\rightarrow$ miR-219-5p mimic

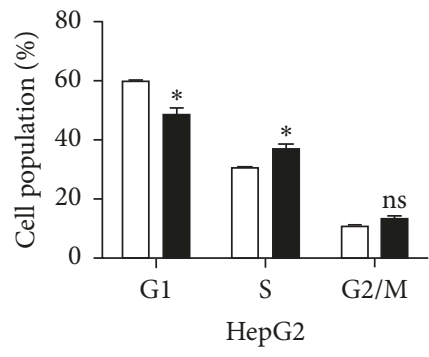

miR-219-5p ctrl miR-219-5p mimic

(b)

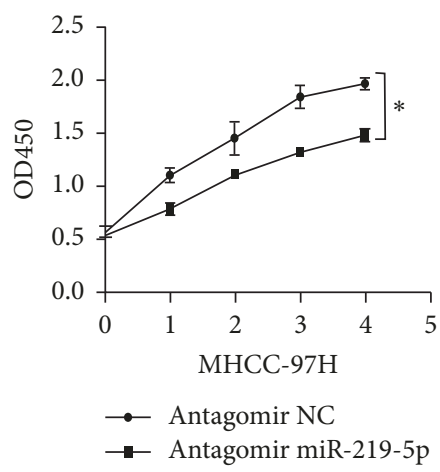

(a)

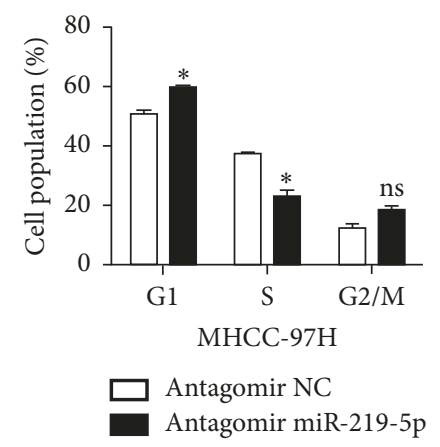

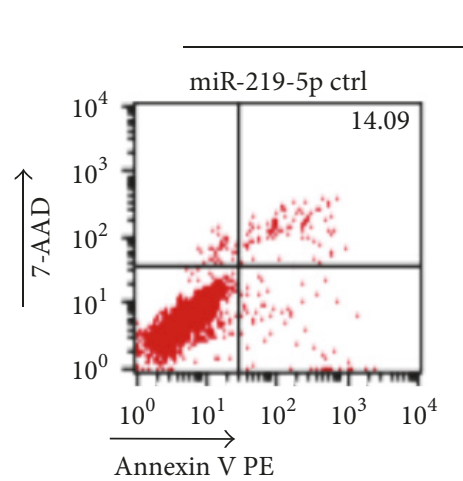

HepG2

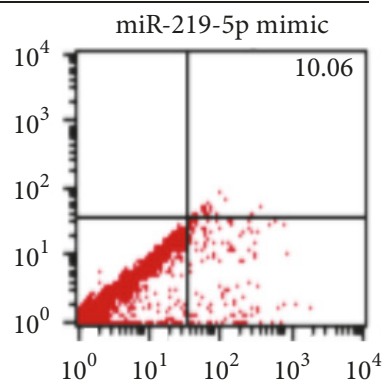

(c)
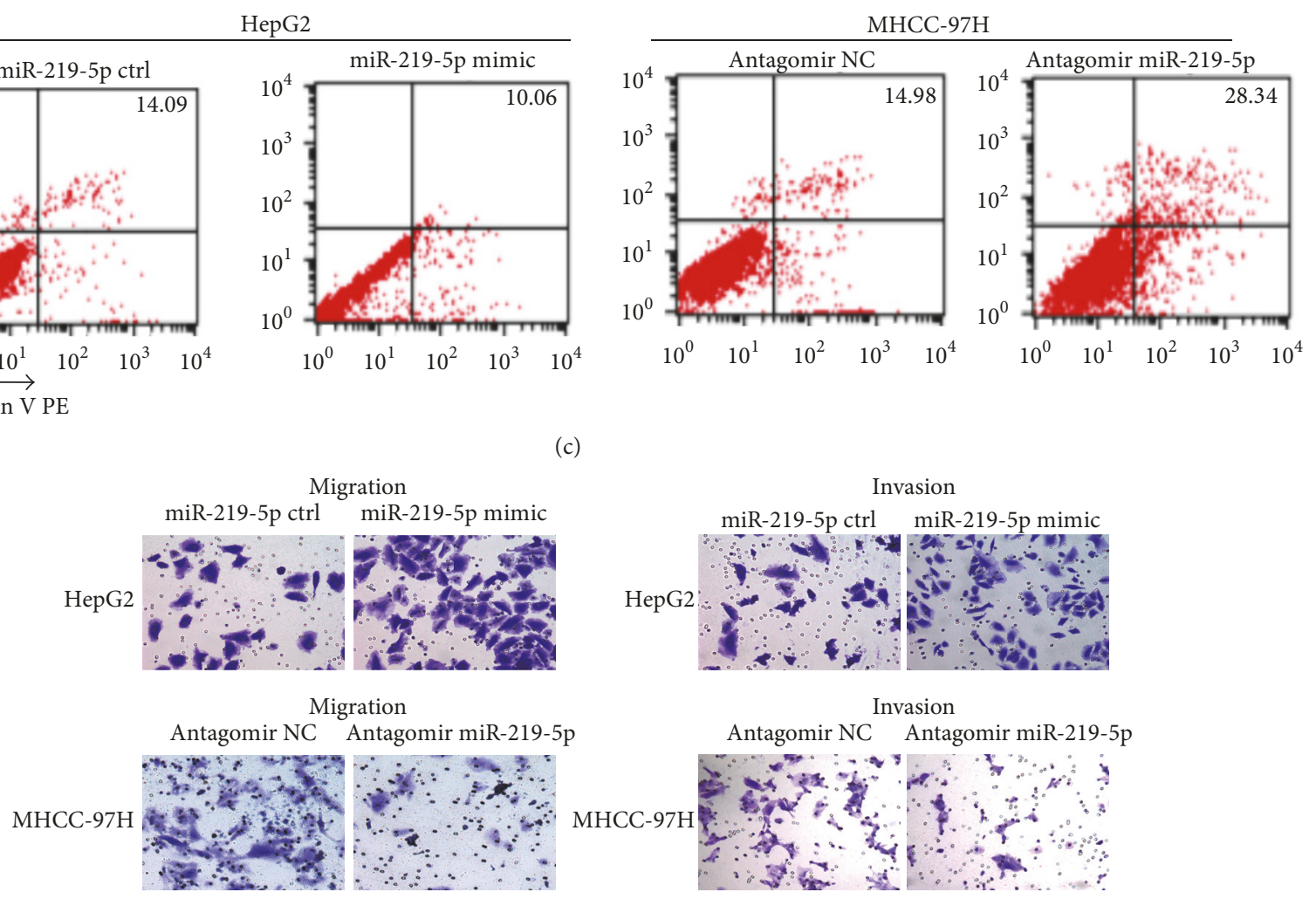

Invasion

Antagomir NC Antagomir miR-219-5p

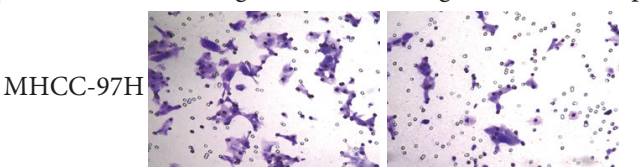

(d)

FIGURE 2: The effects of miR-219-5p on in vitro proliferation and invasion of HCC cells. (a) The alterations in cell proliferation of HepG2 cells after upregulation of miR-219-5p by mimic transfection (left) and MHCC-97H cells after knockdown of miR-219-5p by antagomir (right) were detected by CCK8 assay. (b) The cell cycle distribution of HepG2 and MHCC-97H cells after transfection with miR-219-5p mimic or antagomir. (c) Representative pictures of apoptosis of HepG2 and MHCC-97H cells after transfection with miR-219-5p mimic or antagomir detected by flow cytometry. (d) Migration and invasion of cells were determined by transwell assay in HepG2 and MHCC-97H cells treated with miR-219-5p mimic/antagomir and the corresponding negative control (magnification $\times 100$ ). Data are shown as mean \pm SD. ${ }^{*} P<0.05$, ${ }^{* * *} P<0.001$ versus the control. 


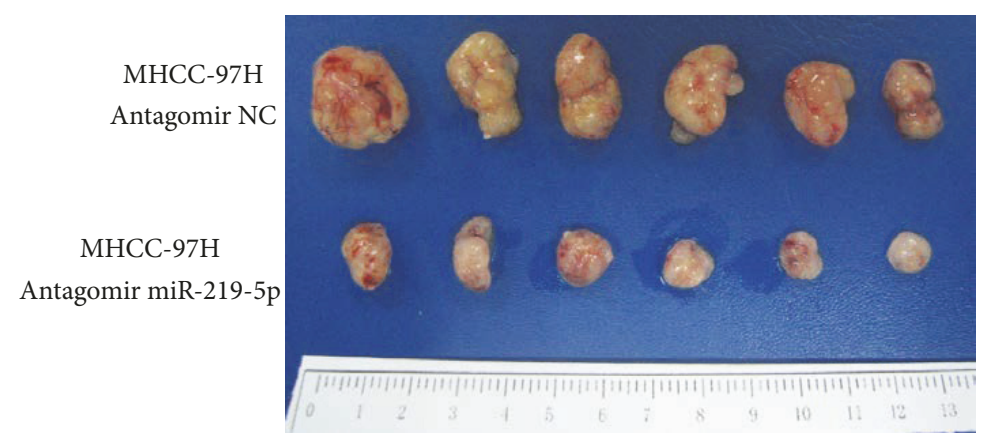

(a)

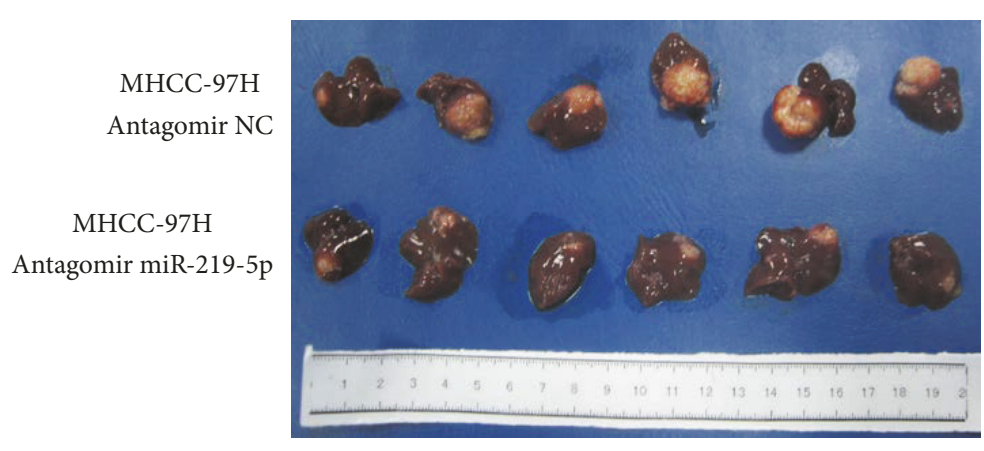

(c)

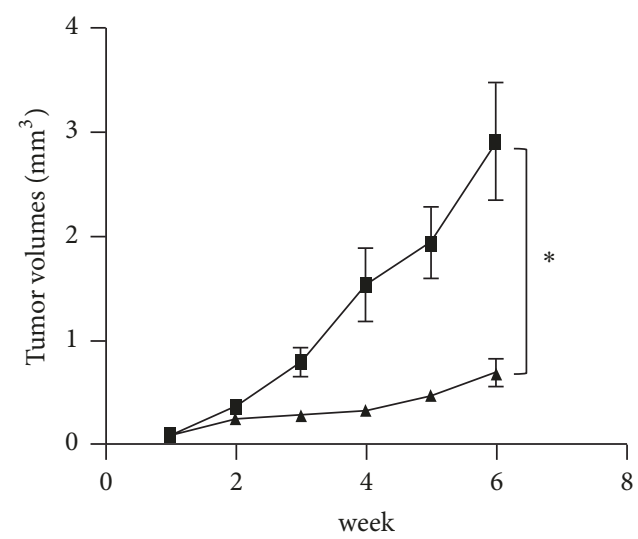

- MHCC-97H-Antagomir NC

$\rightarrow$ MHCC-97H-Antagomir miR-219-5p

(b)

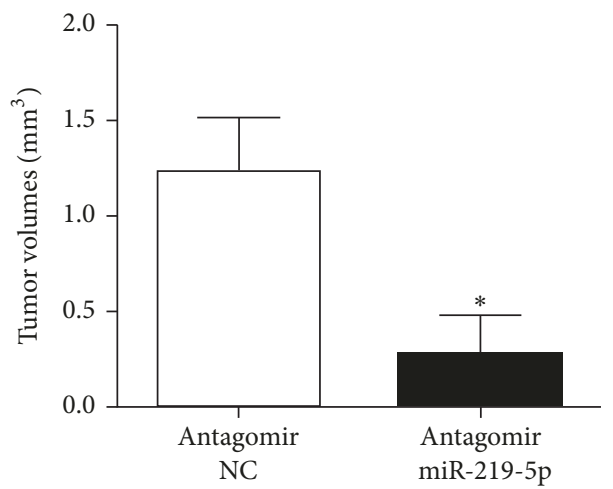

MHCC-97H

Antagomir NC

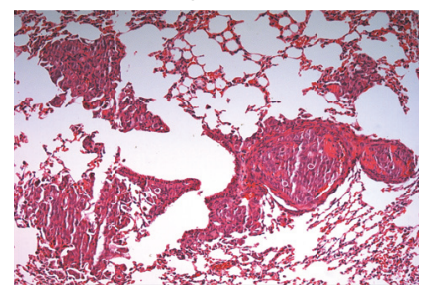

MHCC-97H

Antagomir miR-219-5p

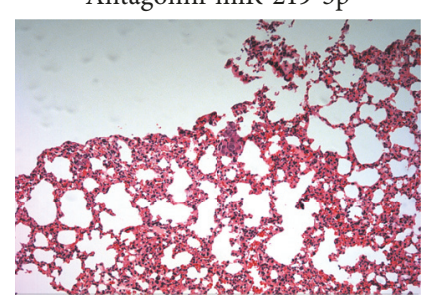

(d)

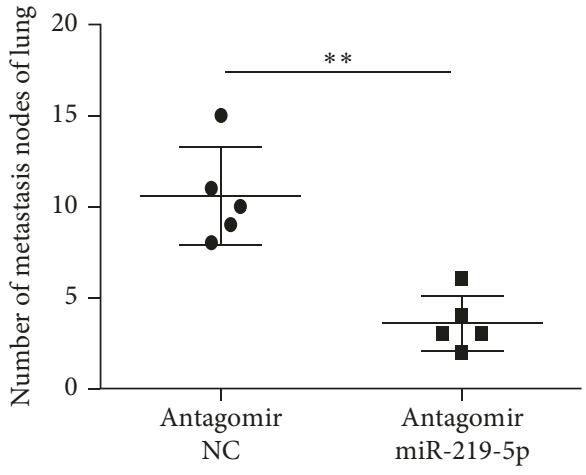

- Antagomir NC

- Antagomir miR-219-5p

FIGURE 3: Effects of miR-219-5p on in vivo tumor growth and lung metastasis of HCC. (a, b) The differences in tumor size and volume of the subcutaneous implantation models of MHCC-97H cells after transfection with miR-219-5p antagomir or antagomir NC. (c) Comparison of the tumor volumes in the orthotopic implantation models of MHCC-97H cells after transfection with antagomir to knockdown miR-219-5p. (d) Representative images of lung metastasis (left) and comparison of the numbers of lung metastatic nodes in orthotopic implantation nude mice models of MHCC-97H cells after transfected with miR-219-5p antagomir or antagomir NC (magnification $\times 200)$. Data are shown as mean \pm SD. ${ }^{*} P<0.05,{ }^{* *} P<0.01$. 


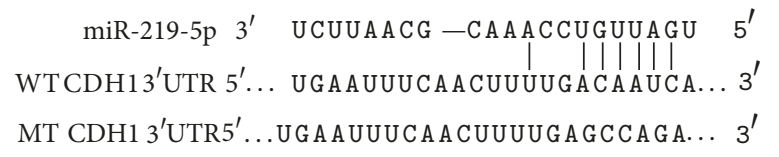

(a)

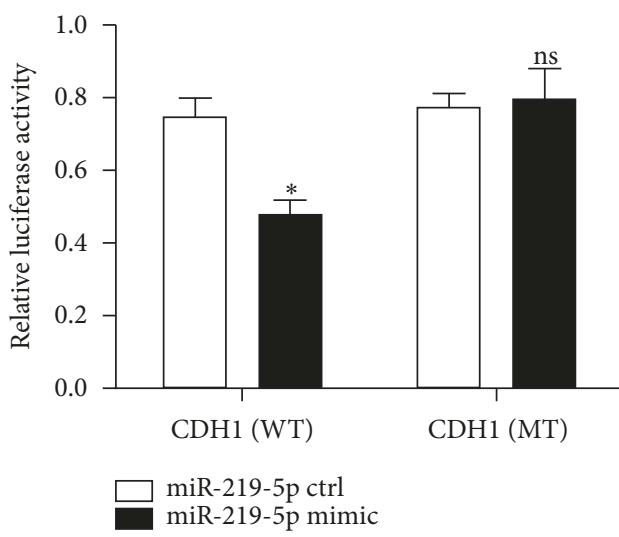

(b)

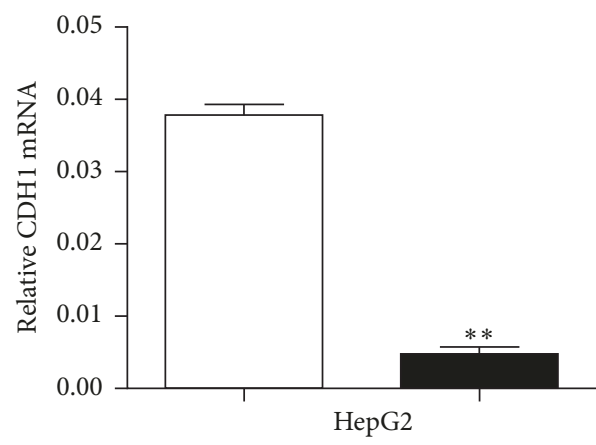

$\square$ miR-219-5p ctrl

miR-219-5p mimic

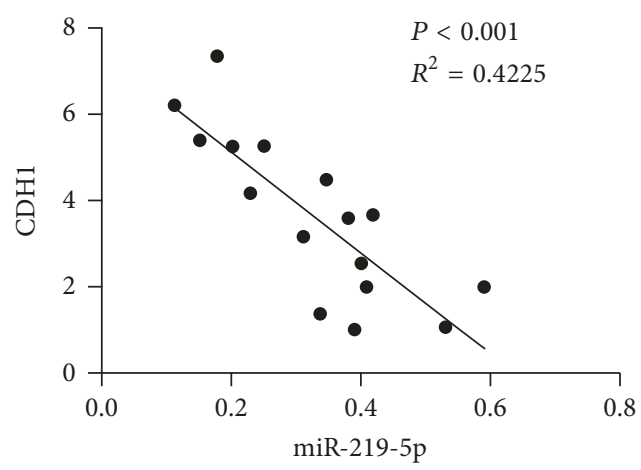

(e)

(d)

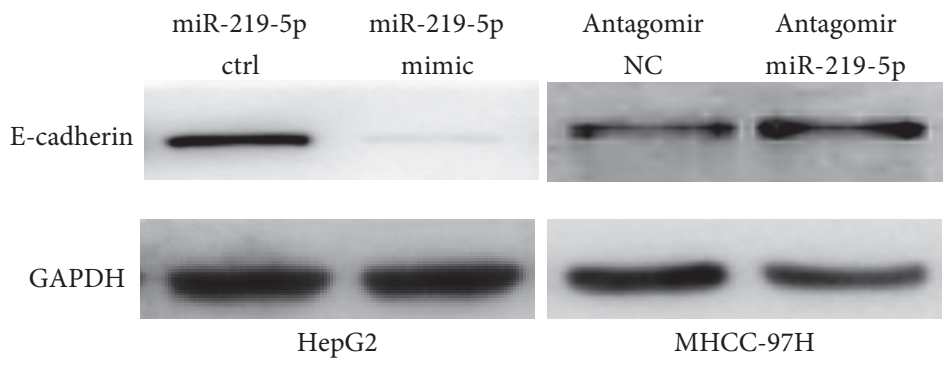

(c)
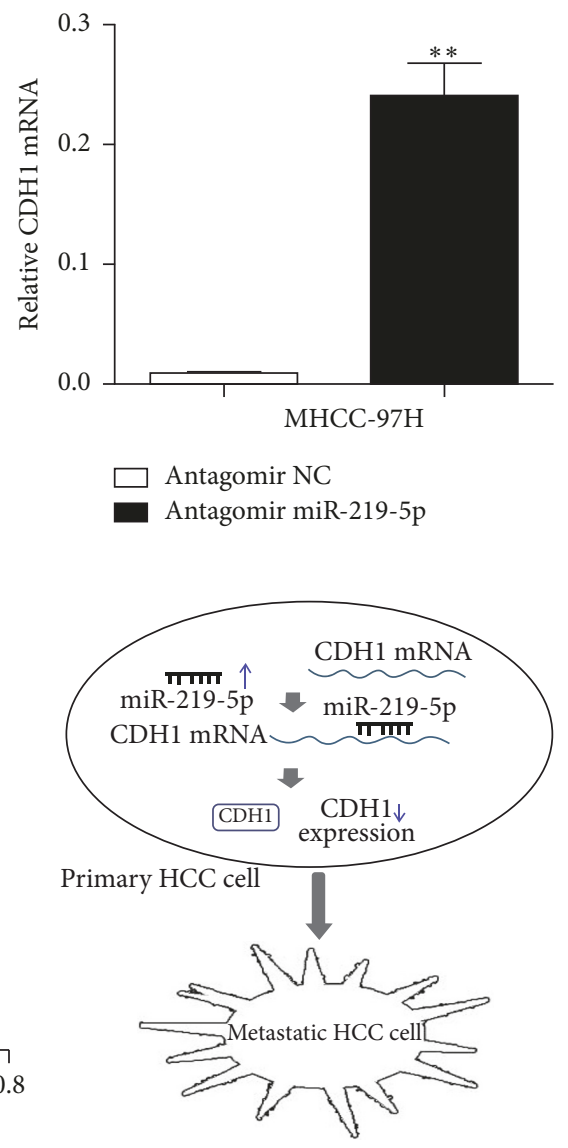

(f)

FIGURE 4: CDH1 is identified as a downstream target of miR-219-5p. (a) Sequences of CDH1 $3^{\prime} \mathrm{UTR}$ and miR-219-5p according to the prediction of microRNA.org. Wild-type and mutated-type binding sequences of CDH1 3'UTR are shown. (b) Relative luciferase activity in HEK293T cells transfected with reporter vector containing wild-type or mutated-type binding sequence along with miR-219-5p mimic or negative control. CDH1 protein (c) and mRNA levels (d) in HCC cells treated with miR-219-5p mimic or antagomir. (e) Linear regression analysis between miR-219-5p and CDH1 in HCCs. (f) Working model for the role of miR-219-5p in regulation of CDH1 in HCC. Data are shown as mean \pm SD. ${ }^{*} P<0.05,{ }^{* *} P<0.01$. 
we found that miR-219-5p could promote in vitro proliferation, migration, and invasion of HCC cells. Furthermore, using loss-functional assays, we demonstrated that miR-219$5 \mathrm{p}$ promoted in vivo tumor growth and distal pulmonary metastasis of HCC. These provided more evidence to support that miR-219-5p is an important promoter for HCC growth and metastasis.

Another significant finding of the present study is that CDH1 is identified as a downstream target of miR-2195 p. $\mathrm{CDH} 1$, a suppressive oncogene, encodes the epithelial cell adhesion molecule, E-cadherin, which contributes to cell polarity and cell-cell adhesion [19-21]. Low expression levels of CDH1 were found to be correlated with aggressive clinicopathological factors and poor survival [22, 23]. Also, CDH1 inactivation resulted in the loss of cell-cell adhesion, which contributes to metastasis in a variety of tumors [24-26]. Furthermore, there is increasing evidence that multiple mechanisms are involved in the expression of $\mathrm{CDH} 1$, including epigenetic DNA methylation, somatic mutations, chromosomal deletions, and protein modification [11, 27, 28]. Recently, miRNAs have been determined to play gene-regulatory roles [28]. In this study, we found the following: (1) Bioinformatic analysis indicated that $\mathrm{CDH} 1$ can be a potential downstream target of miR-219-5p. (2) In a dual-luciferase reporter assay, cotransfection of miR-219$5 \mathrm{p}$ with $\mathrm{CDH} 1$ containing wild-type rather than mutatedtype binding sequence resulted in a significant decrease in luciferase activity. (3) miR-219-5p upregulation led to a significant decrease of CDH1 expression; miR-219-5p knockdown resulted in enhanced CDH1 expression. (4) The linear regression analysis showed a negative relevance between miR-219$5 p$ and CDH1 in HCCs. These indicated that miR-219-5p is closely associated with negative regulation of CDH1 and $\mathrm{CDH} 1$ is a direct target of miR-219-5p. Collectively, miR-2195 p promotes HCC growth and metastasis by downregulating CDH1 (Figure 4(f)).

In conclusion, these data suggest that miR-219-5p upregulation is an independent prognostic indicator for HCC patients. It plays an important role in promoting HCC growth and metastasis by downregulating $\mathrm{CDH}$. These provide more clues to develop novel strategies to combat HCC metastasis.

\section{Conflicts of Interest}

The authors declare that they have no conflicts of interest.

\section{Authors' Contributions}

Jing Yang and Yuan-Yuan Sheng contributed equally as first authors.

\section{Acknowledgments}

This research is supported by Shanghai Municipal Natural Science Foundation (13ZR1402000), National Key Basic Research Program of China (2013CB910500), and the National Key Research and Development Program of China (2017YFC1308604).

\section{Supplementary Materials}

Table S1: the primers listed were used for qPCR. Supplementary Figure 1: (A) miR-219-5p expressions in six liver cancer cell lines. GAPDH served as internal control. Data are shown as mean $+\mathrm{SD} .{ }^{*} P<0.05,{ }^{* *} P<0.01$. Supplementary Figure 2: the relative expression levels of miR-219$5 \mathrm{p}$ in HepG2 and MHCC-97H cells were examined after the cells were treated with miR-219-5p mimic, antagomir, or negative control (A) for $48 \mathrm{~h}$ using RT-qPCR. The analysis of distribution of cells with miR-219-5p mimic/antagomir was shown by histogram (B). The histogram of migration and invasion shows the mean $\pm \mathrm{SD}$ of three independent experiments $(\mathrm{C})$. Data are shown as mean $+\mathrm{SD} .{ }^{*} P<0.05$, ${ }^{* *} P<0.01$. (Supplementary Materials)

\section{References}

[1] R. Siegel, D. Naishadham, and A. Jemal, "Cancer statistics, 2013," CA: A Cancer Journal for Clinicians, vol. 63, no. 1, pp. 11-30, 2013.

[2] A. Villanueva, Y. Hoshida, C. Battiston et al., "Combining clinical, pathology, and gene expression data to predict recurrence of hepatocellular carcinoma," Gastroenterology, vol. 140, no. 5, pp. 1501-1512.e2, 2011.

[3] J. Bruix and J. M. Llovet, "Major achievements in hepatocellular carcinoma," The Lancet, vol. 373, no. 9664, pp. 614-616, 2009.

[4] Y. W. Kong, D. Ferland-McCollough, T. J. Jackson, and M. Bushell, "microRNAs in cancer management," The Lancet Oncology, vol. 13, no. 6, pp. e249-e258, 2012.

[5] S. Giordano and A. Columbano, "MicroRNAs: new tools for diagnosis, prognosis, and therapy in hepatocellular carcinoma?" Hepatology, vol. 57, no. 2, pp. 840-847, 2013.

[6] L. Liang, C.-M. Wong, Q. Ying et al., "MicroRNA-125b suppressesed human liver cancer cell proliferation and metastasis by directly targeting oncogene LIN28B2," Hepatology, vol. 52, no. 5, pp. 1731-1740, 2010.

[7] L. Gramantieri, M. Ferracin, F. Fornari et al., "Cyclin G1 is a target of miR-122a, a MicroRNA frequently down-regulated in human hepatocellular carcinoma," Cancer Research, vol. 67, no. 13, pp. 6092-6099, 2007.

[8] L. Zhao, A. M. Bode, Y. Cao, and Z. Dong, "Regulatory mechanisms and clinical perspectives of miRNA in tumor radiosensitivity," Carcinogenesis, vol. 33, no. 11, pp. 2220-2227, 2012.

[9] R. A. Mathias, S. K. Gopal, and R. J. Simpson, "Contribution of cells undergoing epithelial-mesenchymal transition to the tumour microenvironment," Journal of Proteomics, vol. 78, pp. 545-557, 2013.

[10] A. Budhu, H. L. Jia, M. Forgues et al., "Identification of metastasis-related microRNAs in hepatocellular carcinoma," Hepatology, vol. 47, no. 3, pp. 897-907, 2008.

[11] G. Berx, K.-F. Becker, H. Höfler, and F. Van Roy, "Mutations of the human E-cadherin (CDH1) gene," Human Mutation, vol. 12, no. 4, pp. 226-237, 1998.

[12] L.-X. Qin and Z.-Y. Tang, "The prognostic molecular markers in hepatocellular carcinoma," World Journal of Gastroenterology, vol. 8, no. 3, pp. 385-392, 2002.

[13] K. Kai, R. L. Dittmar, and S. Sen, "Secretory microRNAs as biomarkers of cancer," Seminars in Cell \& Developmental Biology, 2017. 
[14] M. B. Afonso, P. M. Rodrigues, A. L. Simão, and R. E. Castro, "Circulating microRNAs as potential biomarkers in non-alcoholic fatty liver disease and hepatocellular carcinoma," Journal of Clinical Medicine, vol. 5, no. 3, 2016.

[15] K. Han, J. Li, H. Zhao et al., "Identification of the typical miRNAs and target genes in hepatocellular carcinoma," Molecular Medicine Reports, vol. 10, no. 1, pp. 229-235, 2014.

[16] M. Milazzo, F. Fornari, and L. Gramantieri, "MicroRNA and hepatocellular carcinoma: Biology and prognostic significance," Minerva Gastroenterologica e Dietologica, vol. 57, no. 3, pp. 257271, 2011.

[17] C. Li, J. Dong, Z. Han, and K. Zhang, "MicroRNA-219-5p Represses the Proliferation, Migration, and Invasion of Gastric Cancer Cells by Targeting the LRH-1/Wnt/ $\beta$-Catenin Signaling Pathway," Oncology Research : Featuring Preclinical and Clinical Cancer Therapeutics, vol. 25, no. 4, pp. 617-627, 2017.

[18] Q. Wang, L. Zhu, Y. Jiang, J. Xu, F. Wang, and Z. He, "miR-219$5 p$ suppresses the proliferation and invasion of colorectal cancer cells by targeting calcyphosin," Oncology Letters, vol. 13, no. 3, pp. 1319-1324, 2017.

[19] B. M. Gumbiner, "Regulation of cadherin-mediated adhesion in morphogenesis," Nature Reviews Molecular Cell Biology, vol. 6, no. 8, pp. 622-634, 2005.

[20] M. Yilmaz and G. Christofori, "EMT, the cytoskeleton, and cancer cell invasion," Cancer and Metastasis Reviews, vol. 28, no. 1-2, pp. 15-33, 2009.

[21] S. Lamouille, J. Xu, and R. Derynck, "Molecular mechanisms of epithelial-mesenchymal transition," Nature Reviews Molecular Cell Biology, vol. 15, no. 3, pp. 178-196, 2014.

[22] Y. Wei, J. T. Van Nhieu, S. Prigent, P. Srivatanakul, P. Tiollais, and M.-A. Buendia, "Altered expression of E-cadherin in hepatocellular carcinoma: Correlations with genetic alterations, $\beta$ catenin expression, and clinical features," Hepatology, vol. 36, no. 3, pp. 692-701, 2002.

[23] X.-M. Jiang, J.-B. Zhang, J. Xiong, X.-X. Huang, and Z.-G. Ren, "Altered distribution and expression pattern of e-cadherin in hepatocellular carcinomas: Correlations with prognosis and clinical features," Asian Pacific Journal of Cancer Prevention, vol. 13, no. 12, pp. 6455-6461, 2012.

[24] T. T. Onder, P. B. Gupta, S. A. Mani, J. Yang, E. S. Lander, and R. A. Weinberg, "Loss of E-cadherin promotes metastasis via multiple downstream transcriptional pathways," Cancer Research, vol. 68, no. 10, pp. 3645-3654, 2008.

[25] K. Vleminckx, L. Vakaet Jr., M. Mareel, W. Fiers, and F. Van Roy, "Genetic manipulation of E-cadherin expression by epithelial tumor cells reveals an invasion suppressor role," Cell, vol. 66, no. 1, pp. 107-119, 1991.

[26] G. Giannelli, P. Koudelkova, F. Dituri, and W. Mikulits, "Role of epithelial to mesenchymal transition in hepatocellular carcinoma," Journal of Hepatology, vol. 65, no. 4, pp. 798-808, 2016.

[27] J. Paredes and et al., "Epithelial E- and P-cadherins: role and clinical significance in cancer," Biochimica et Biophysica Acta, no. 2, pp. 297-311, 1826.

[28] T. Maretzky, K. Reiss, A. Ludwig et al., "ADAM10 mediates Ecadherin shedding and regulates epithelial cell-cell adhesion, migration, and $\beta$-catenin translocation," Proceedings of the National Acadamy of Sciences of the United States of America, vol. 102, no. 26, pp. 9182-9187, 2005. 


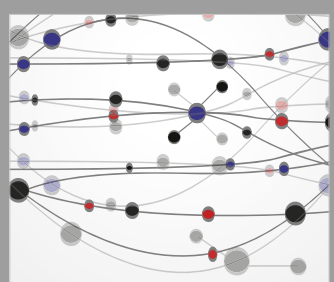

The Scientific World Journal
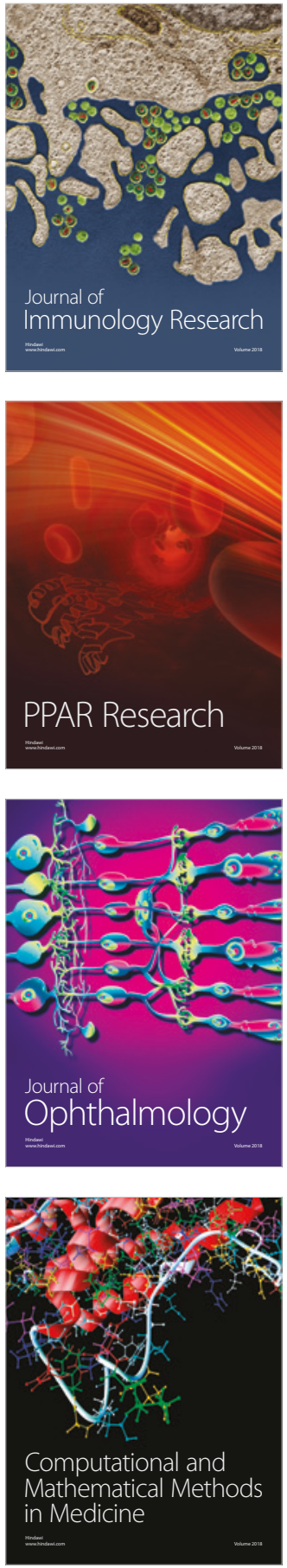

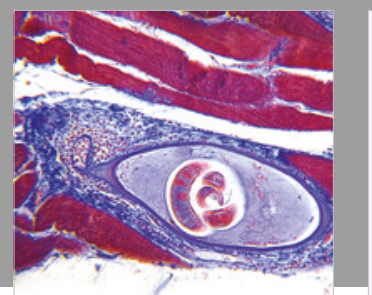

Gastroenterology Research and Practice

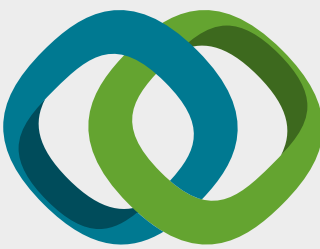

\section{Hindawi}

Submit your manuscripts at

www.hindawi.com
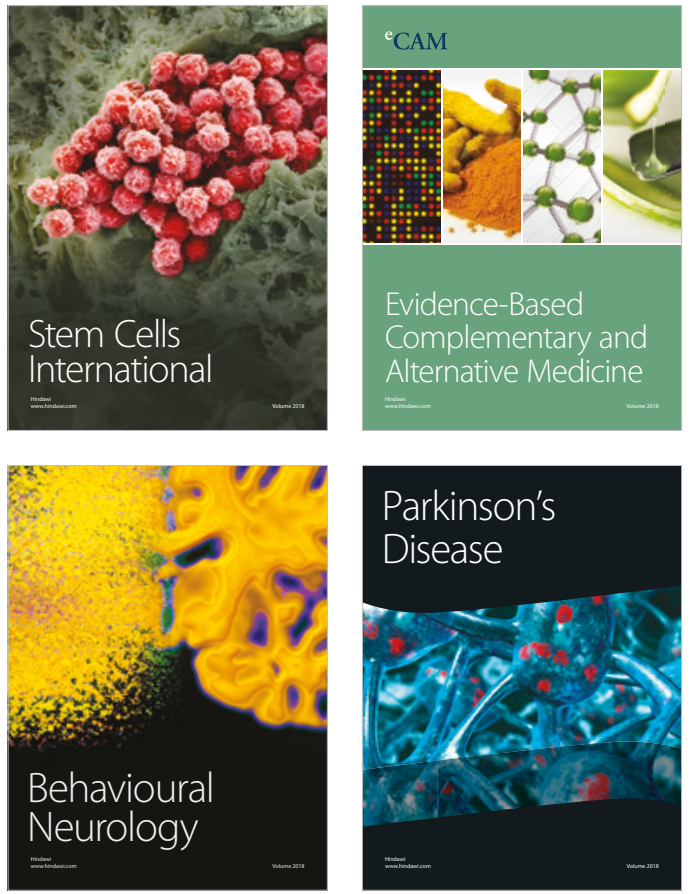

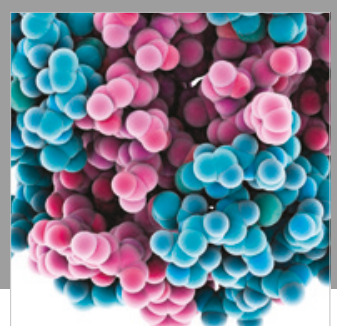

ournal of

Diabetes Research

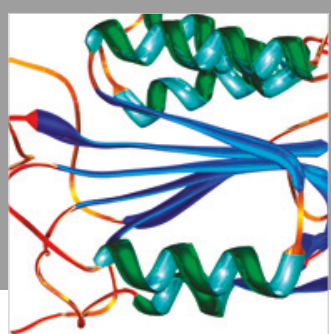

Disease Markers
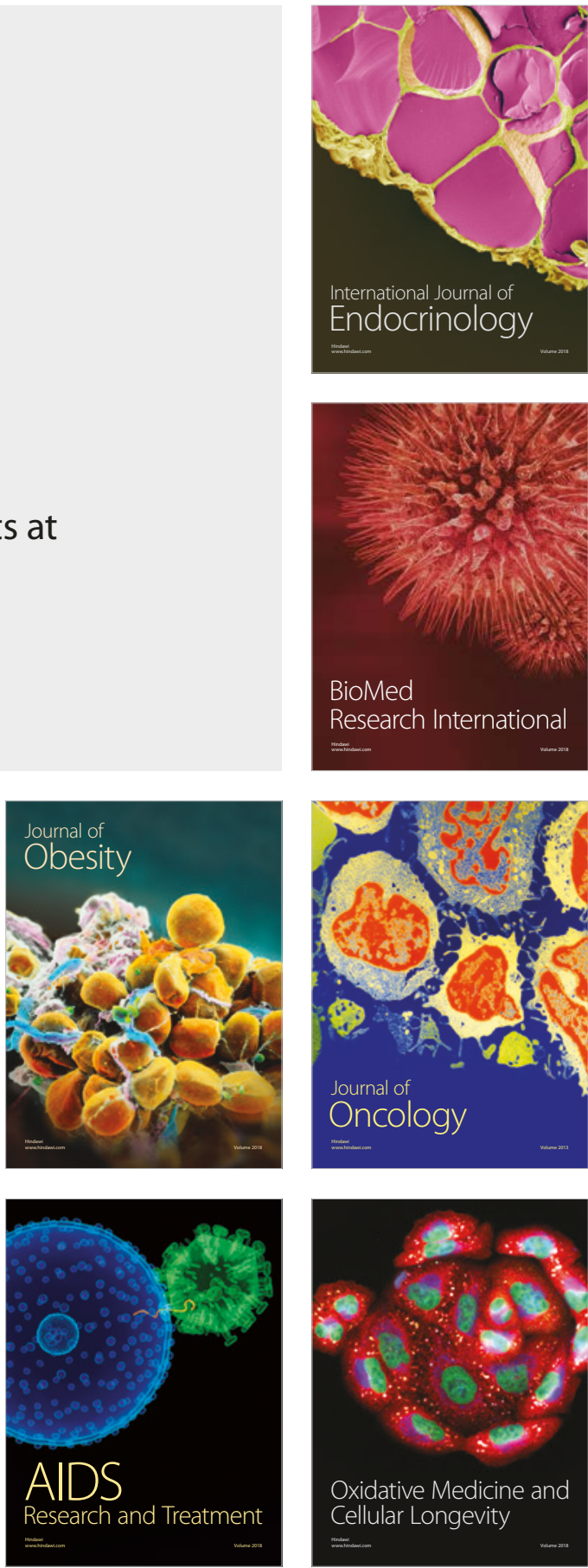